\title{
The 2014 American Heart Association/American College of Cardiology guideline for the management of patients with valvular heart disease: A changing landscape
}

\author{
Robert C. Neely, MD, Marzia Leacche, MD, Igor Gosev, MD, Tsuyoshi Kaneko, MD, \\ John G. Byrne, MD, FACC, and Michael J. Davidson, MD
}

The American Heart Association (AHA) and American College of Cardiology (ACC) have updated the practice guidelines for the treatment of patients with valvular heart disease (VHD). ${ }^{1}$ The writing committee and task force members were experts in all aspects of valvular disease and included representatives from the Society of Cardiovascular Anesthesiologists, Society for Cardiovascular Angiography and Interventions, American Society of Echocardiography, and surgical representatives from The American Association for Thoracic Surgery and Society of Thoracic Surgery (STS). Importantly, the writing committee members were free of industry affiliations.

The original AHA/ACC VHD guidelines were released in 1998, revised in 2006, and updated in $2008 .^{2}$ The recent growth in therapeutic options for VHD requires the incorporation of evolving techniques and current evidence to determine best practice. With the expansion of transcatheter and minimally invasive valve surgery, the question is not simply should we intervene, but how and when. To outline concomitant nonsurgical therapeutic advances, the Task Force coined the phrase guideline-directed medical therapy "to represent optimal medical therapy as defined by [the] ACC/AHA guideline (primarily class I)-recommended therapies." ${ }^{11}$ Thus, the current guidelines reflect updates in medical and surgical advances, with new sections summarizing the role of transcatheter aortic valve replacement (TAVR) and transcatheter approaches for the mitral valve. Evolving approaches for aortic aneurysms and valve type choices are also discussed.

For severe aortic stenosis, surgical AVR remains a class I, level of evidence A, recommendation for low or intermediate surgical risk candidates meeting the criteria for valve replacement. Drawing from several large, multiinstitutional, randomized controlled trials, the guidelines state that "TAVR is recommended in patients who meet an indication for AVR who have a prohibitive risk for

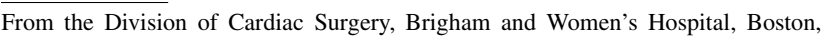
Mass.

Disclosures: Authors have nothing to disclose with regard to commercial support. Received for publication May 8, 2014; accepted for publication May 8, 2014.

Address for reprints: John G. Byrne, MD, FACC, Division of Cardiac Surgery, Brigham and Women's Hospital, 75 Francis St, Boston, MA 02115 (E-mail: jbyrne@ partners.org).

J Thorac Cardiovasc Surg 2014;148:5-6

$0022-5223 / \$ 36.00$

Copyright (c) 2014 by The American Association for Thoracic Surgery

http://dx.doi.org/10.1016/j.jtcvs.2014.05.015
}

surgical AVR and predicted post-TAVR survival greater than 12 months" (class I, level of evidence B). In the modern era, patients must be risk stratified by comorbidities, procedure complexity, and frailty measures to determine which intervention, if any, is most appropriate. In addition to the traditional STS predicted risk of mortality, frailty estimates have been incorporated to reflect the spectrum of a patient's preoperative functional status: no frailty, able to perform 1 activity of daily living and perform a 5-m walk in $<6$ seconds; mild degree of frailty, unable to perform 1 activity of daily living and perform a 5 -m walk in $<6$ seconds; and moderate-to-severe degree of frailty, unable to perform $\geq 2$ activities of daily living. ${ }^{1}$ Candidates suitable for TAVR must be within 1 of 2 categories:

High-risk-STS predicted risk of mortality $>8 \%$ or $\geq 2$ indexes of frailty or $>2$ organ systems compromised or possible procedure-specific impediment (eg, severely atherosclerotic aorta or a hazardous redo sternotomy with a previous left internal mammary artery conduit crossing the sternum).

Prohibitive risk-predicted risk with surgery or major comorbidity $>50 \%$ at 1 year or $>3$ organ systems compromised or severe procedure-specific impediments (eg, severe atherosclerotic aorta, chest malformations, previous radiotherapy, or previous bypass graft adherent to the sternum).

The Placement of Aortic Transcatheter Valve study demonstrated that among patients deemed at prohibitive risk, TAVR does not appear to benefit those with an STS score of $\geq 15 \%$ (hazard ratio, $0.77 ; 95 \%$ confidence interval, $0.46-1.28 ; P=.31){ }^{3}$ The Task Force summarized that a "poor outcome after TAVR is associated with advanced age, frailty, smoking or chronic obstructive pulmonary disease, pulmonary hypertension, liver disease, prior stroke, anemia, and other systemic conditions. TAVR is not recommended in patients with (1) [a] life expectancy of $<1$ year, even with a successful procedure, and (2) those with a chance of 'survival with benefit' of $<25 \%$ at 2 years." 1 The role of a "heart valve team," therefore, is essential in the evaluation of high-risk surgical candidates. Heart valve Centers of Excellence and designated teams of specialists provide expertise for complex VHD. Given the multiple therapeutic options available for VHD 
in an increasingly older patient population, teams composed of cardiologists, cardiac surgeons, imaging specialists, and other dedicated providers will facilitate a comprehensive patient evaluation and offer expertise in all treatment modalities not available in most hospitals.

We anticipate more information to emerge about the use of TAVR for intermediate-risk candidates. Several studies are ongoing, and, at the time of this writing (May 2014), the evidence is not sufficient to support a change in the guidelines for this population. As this information becomes available and as more data emerge on second- and thirdgeneration devices, the recommendations for the use of TAVR are certain to evolve.

The 2014 guidelines are the first to delineate a role for transcatheter approaches for the mitral valve: "Transcatheter mitral valve repair may be considered for severely symptomatic patients (NYHA [New York Heart Association] class III to IV) with chronic severe primary MR [mitral regurgitation] who have favorable anatomy for the repair procedure and a reasonable life expectancy but who have a prohibitive surgical risk because of severe comorbidities and remain severely symptomatic despite optimal GDMT [guideline-directed medical therapy] for [heart failure]" (class IIB, level of evidence B). To date, only 1 randomized control trial has compared surgical mitral repair and percutaneous mitral valve repair (MitraClip, Abbott Vascular, Santa Clara, Calif). ${ }^{4}$ The percutaneous repair group had a greater incidence of residual mitral regurgitation but demonstrated decreased mitral regurgitation severity, favorable left ventricle remodeling, and improved symptoms. Proper patient selection remains essential and should be performed by a multidisciplinary team, with repair only considered for chronic, primary mitral regurgitation with New York Heart Association class III or IV heart failure symptoms.

In addition to transcatheter techniques, the current guidelines update the size recommendations for aortic aneurysm intervention in the setting of a bicuspid aortic valve. Previous guidelines recommended surgery for ascending aorta dilatation at any level $>5.0 \mathrm{~cm}$ for patients with a bicuspid aortic valve, regardless of valve function. The evidence for such treatment has largely been anecdotal and limited to 2 large, long-term retrospective cohort studies of patients with bicuspid aortic valves. ${ }^{5,6}$ In 1 of these studies, which followed up 416 patients for a mean of 16 years, the incidence of aortic dissection was low and estimated at 3.1 cases $/ 10,000$ patient-years $(95 \%$ confidence interval, $0.5-9.5){ }^{6}$ Thus, surgery is recommended for aortic dilatation of 5.1 to $5.5 \mathrm{~cm}$ only if the patient has a family history of aortic dissection or rapid progression of dilatation $(\geq 0.5$ $\mathrm{cm} / \mathrm{y}$ ). For patients with a bicuspid aortic valve and severe aortic stenosis or regurgitation who are undergoing aortic valve surgery, the threshold for concomitant ascending aorta replacement remains $4.5 \mathrm{~cm}$. For all other circumstances, an operation is indicated for more severe dilatation $(>5.5 \mathrm{~cm})$. These measurements apply to the level of the aortic sinus or any level along the ascending aorta and should not be adjusted for body size.

Finally, the AHA/ACC task force emphasized that patient choice regarding the type of valve prosthesis should be tailored to the individual's lifestyle and decided after an in-depth discussion among patient, surgeon, and cardiologist: "specifically, the tradeoff between the risk of reoperation for bioprosthetic valve degeneration and the risk associated with long-term anticoagulation should be discussed in detail." "At the patient's request, with current evidence, it is reasonable to proceed with a bioprosthetic valve at any age. This is especially true with the potential for subsequent transcatheter valve-in-valve implantation for patients who choose a biologic aortic valve. In contrast, for patients without a contraindication for anticoagulation who are considering a mechanical prosthesis, the guidelines have lowered the recommendation for the age cutoff from 65 years to 60 years for both aortic and mitral valve replacement. ${ }^{1}$

The authors of the guidelines have attempted to develop guidelines to address the clinical circumstances for most patients. Ultimately, appropriate therapy must be tailored to the individual patient and directed by providers working within their area of expertise. With the myriad technological advances since the last published guidelines, it is reasonable to expect continued growth in the field of VHD, with regional heart valve Centers of Excellence playing an ever increasing role. A multidisciplinary approach by experts will help determine when to adhere to these guidelines and when a patient-centered departure is appropriate amid this changing landscape of VHD.

\section{References}

1. Nishimura RA, Otto CM, Bonow RO, Carabello BA, Erwin JP III, Guyton RA, et al. 2014 AHA/ACC Guideline for the Management of Patients With Valvular Heart Disease. J Am Coll Cardiol. 2014; http://dx.doi.org/10.1016/ j.jacc.2014.02.536.

2. Bonow RO, Carabello BA, Chatterjee K, de Leon AC Jr, Faxon DP, Freed MD, et al. 2008 Focused update incorporated into the ACC/AHA 2006 guidelines for the management of patients with valvular heart disease: a report of the American College of Cardiology/American Heart Association Task Force on Practice Guidelines (Writing Committee to revise the 1998 guidelines for the management of patients with valvular heart disease). Endorsed by the Society of Cardiovascular Anesthesiologists, Society for Cardiovascular Angiography and Interventions, and Society of Thoracic Surgeons. J Am Coll Cardiol. 2008;52: e1-142.

3. Kodali SK, Williams MR, Smith CR, Svensson LG, Webb JG, Makkar RR, et al. Two-year outcomes after transcatheter or surgical aortic-valve replacement. N Engl J Med. 2012;366:1686-95.

4. Mauri L, Foster E, Glower DD, Apruzzese P, Massaro JM, Herrmann HC, et al. 4-Year results of a randomized controlled trial of percutaneous repair versus surgery for mitral regurgitation. J Am Coll Cardiol. 2013;62:317-28.

5. Tzemos N, Therrien J, Yip J, Thanassoulis G, Tremblay S, Jamorski MT, et al. Outcomes in adults with bicuspid aortic valves. JAMA. 2008;300:1317-25.

6. Michelena HI, Desjadins VA, Avierinos JF, Russo A, Nkomo VT, Sundt TM, et al. Natural history of asymptomatic patients with normally functioning or minimally dysfunctional bicuspid aortic valve in the community. Circulation. 2008;117: 2776-84. 